\title{
Cystic Endometrial Hyperplasia
}

National Cancer Institute

\section{Source}

National Cancer Institute. Cystic Endometrial Hyperplasia. NCI Thesaurus. Code C34519.

Benign endometrial hyperplasia characterized by the presence of cystically dilated glands. 